\title{
Effect of Exercise Training on the Expression of p53 and iNOS in the Cardiac Muscle of Type I Diabetic Rats
}

\author{
Muhammed Al-Jarrah ${ }^{\mathrm{a}, \mathrm{d}}$, Mohammed Bani Ahmad ${ }^{\mathrm{b}}$, Mikhled Maayah $^{\mathrm{a}}$, Ahed Al-Khatib ${ }^{\mathrm{c}}$
}

\begin{abstract}
Background: Diabetes is associated with a marked increase in the risk of cardiac diseases, including atherosclerosis, cardiomyopathy, and impaired ventricular function. The ability of exercise to reduce the risk of cardiac troubles has been established, but no studies have examined this link in type 1 diabetes. A randomized, controlled animal study was designed using a standard rat model of type 1 diabetes. The goal of this study was to investigate the expression of P53 and iNOS in cardiac muscle of rat with Alloxan-induced diabetes.
\end{abstract}

Methods: Forty rats were randomized into four groups: sedentary control, exercise control, sedentary or exercised diabetic. Diabetes was induced by alloxan injection. After the development of diabetes, a 4-week treadmill training program was initiated. Cardiac muscle left ventricle was evaluated for the expression of p53 and iNOS using immunohistochemistry.

Results: Both levels of iNOS and p53 increased in the heart of diabetic rats. The expression level of p53 increased significantly in the diabetic sedentary group compared to sedentary control groups $(\mathrm{P}<$ $0.02)$. Exercise training significantly decreased the expression level of $\mathrm{p} 53(\mathrm{P}<0.005)$, but the decrease level of iNOS in the exercised diabetic groups was not significant $(\mathrm{P}<0.07)$.

Conclusions: Diabetes leads to harmful effects on the cardiac muscle. Exercise training decreases in part the complications of diabetes in the heart of type I diabetes.

\footnotetext{
Manuscript accepted for publication October 5, 2012

${ }^{\mathrm{a} D e p a r t m e n t ~ o f ~ R e h a b i l i t a t i o n ~ S c i e n c e s, ~ J o r d a n ~ U n i v e r s i t y ~ o f ~ S c i e n c e ~}$ and Technology (JUST). 22110 Irbid, Jordan

${ }^{\mathrm{b}}$ Department of Medical Laboratory Sciences, JUST, Jordan

${ }^{\mathrm{c}}$ Department of Forensic Sciences and Toxicology, JUST, Jordan

${ }^{\mathrm{d}}$ Corresponding author: Muhammed Al-Jarrah, Department of

Rehabilitation Sciences, Faculty of Applied Medical Sciences, Jordan

University of Science and Technology, 22110 Irbid P O Box 3030

Jordan. Email: jarrahm@just.edu.jo

The corresponding author is in a leave to Fatima College of Health

Sciences, UAE. Email: Muhammed.Aljarrah@ffchs.ac.ae

doi: http://dx.doi.org/10.4021/jem123e
}

Keywords: Type I diabetes; p53; iNOS; Cardiac muscle

\section{Introduction}

It is well known that patients with diabetes have increased risk of cardiovascular diseases [1]. In type 1 diabetes, the heart is subject to a variety of pathological insults, including atherosclerosis, cardiac autonomic neuropathy, and possibly intrinsic cardiomyopathy [2]. Moreover, people suffering from diabetes are more likely to be at risk for heart attacks, strokes and high blood pressure. Vascular problems, such as poor circulation to the legs and feet, are also more likely to affect diabetes patients.

In the streptrozotocin treated rats there is a cardiomyopathy, which is characterized by reduced contractility force, relaxation time, and cardiac work [3]. Diabetic cardiomyopathy is a common complication leading to heightened risk of heart failure and death [4]. Excess reactive oxygen species (ROS) have been widely implicated in diabetes and its many complications [5]. The heart contains low levels of antioxidants, making it prone to oxidative damage [6]. It has been shown that diabetic hearts display increased levels of lipid peroxidation and induction of antioxidant enzymes, indicating the involvement of oxidative stress in this disease [7]. While the association between glycemia and microvascular complication is already established, there have been very few studies that clarified the beneficial effects of exercise on the heart of type 1 diabetics [8].

Both types of diabetes are independent risk factors for cardiovascular diseases. Moreover, myocardial ischemia due to coronary atherosclerosis commonly occurs without symptoms in diabetic patients $[9,10]$. As a result, multivessel atherosclerosis is usually present before ischemic symptoms occur and before treatment is planned. A delayed recognition of various forms of cardiovascular diseases may worsen the complications and prognosis in diabetic patients.

Nitric oxide (NO) and its isoforms, inducible, neuronal, and endothelial is an important signaling molecule that acts in many tissues to regulate a diverse range of physiological 


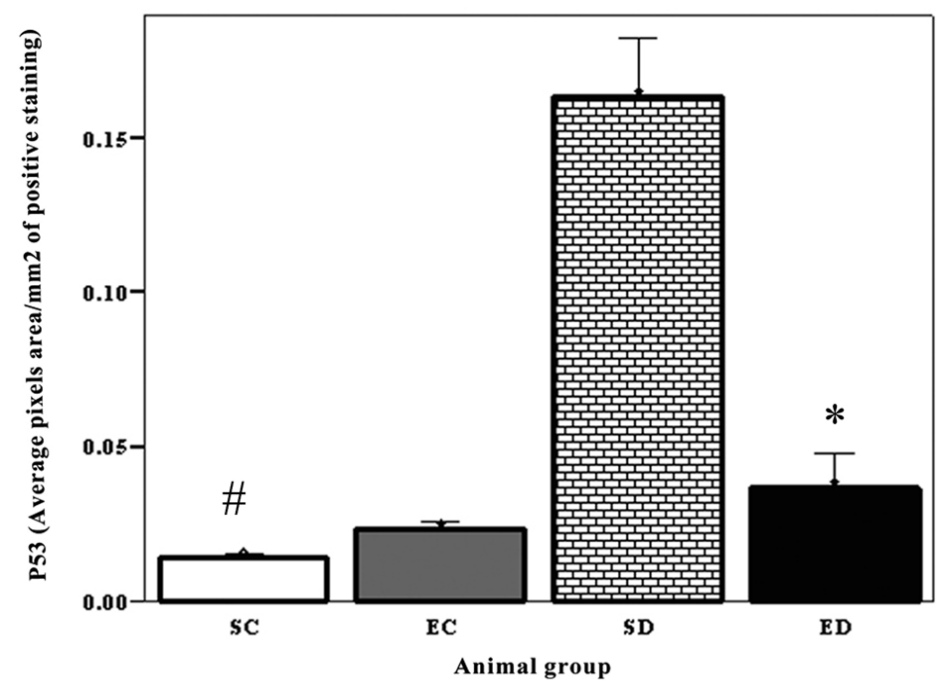

Figure 1. Expression of P53 in the cardiac muscle. The expression level of p53 increased significantly in the diabetic sedentary group compared to sedentary control groups $(P<0.02$, \#). Exercise training significantly decreased the expression level of P53 (P < 0.005), SC: Sedentary Control. EC: Exercised Control. SD: Sedentary Diabetic. ED: Exercise Diabetic.

and cellular processes. Because of its importance in vascular function, abnormal production of $\mathrm{NO}$, as occurs in different disease states such as diabetes and heart failure, can adversely affect blood flow and other vascular functions [11].

P53 is a tumor suppressor gene known to promote cardiac dysfunction in both type 1 and type 2 Diabetes [12]. When activated, p53 can result in cell cycle arrest [13].The role of exercise on the heart function is not well investigated in the literature; the main goal of this study was to examine the effect of exercise on the expression of p53 and iNOS in the heart of rats with induced type 1diabetes.

\section{Materials and Methods}

\section{Animals}

Forty Sprague-Dawley rats were used in this study and randomly assigned into one of four groups. Sedentary control ( $S C, n=10)$, exercise control $(E C, n=10)$, sedentary diabetic $(S D, n=10)$, exercise diabetic $(E D, n=10)$. Animals were housed in individual cages at a $22 \pm 1{ }^{\circ} \mathrm{C}$ in a controlled room with a 12:12 light: dark cycle. The animals were allowed free access to standard chow and water. Animal care and experiments were performed in accordance with Jordan University of Science and Technology research committee guidelines for animal experimentation. Rats in the two diabetic groups received an intraperitoneal injection of alloxan $(120 \mathrm{mg} / \mathrm{kg})$ or saline in the control groups. Three days later, a confirmation of successful induction of diabetes was assessed by demonstration of hyperglycemia in the rats. Animals with fasting blood glucose above $250 \mathrm{mg} / \mathrm{dL}$ were classified as diabetic animals.

\section{Exercise Protocol}

The exercise training protocol utilized was based on previous publications providing evidence of adequate systemic and cellular adaptations with this level of aerobic exercise [14]. Aerobic exercise training was conducted on a custom treadmill with 8 separate lanes. Following the alloxan or saline treatment, the rats in the exercised groups were introduced to the treadmill slowly over the course of a week with initial orientation and walking on the moving treadmill. The 4 week exercise protocol did not begin until rats could run at a speed of $18 \mathrm{~m} / \mathrm{min}$. The training protocol was individualized for each animal, but in general consisted of running for $40 \mathrm{~min} /$ day for 5 days/week at a speed of $18 \mathrm{~m} / \mathrm{min}$. Sedentary rats did not exercise; however, they were transported daily to the training room so that they were exposed to the same environment as the exercised groups of animals.

\section{Immunostaining of p53 and iNOS in the heart}

Tissues were collected from the left ventricle of the heart and fixed in $4 \%$ parafolmaldehyde and subsequently embedded in paraffin. Sections $(5 \mu \mathrm{m})$ were mounted on coated slides (microslides sc-24976, Santa Cruz). Prior to staining, samples were deparaffinized in xylene twice for 2 minutes, then rehydrated through serial dilutions of alcohol $(100 \%, 90 \%$, $80 \%$, and $70 \%$ ) ending in water ( 2 minutes each step). This was followed by treatment under pressure in the reveal solution (RV1000M, Biocare Medical, Concord, CA) for 2 minutes in the Decloaking chamber (Biocare Medical) in order to retrieve the antigens, and later to block endogenous biotin. After cooling to room temperature, sections were incubated with $3 \%$ hydrogen peroxide in methanol in order to block 


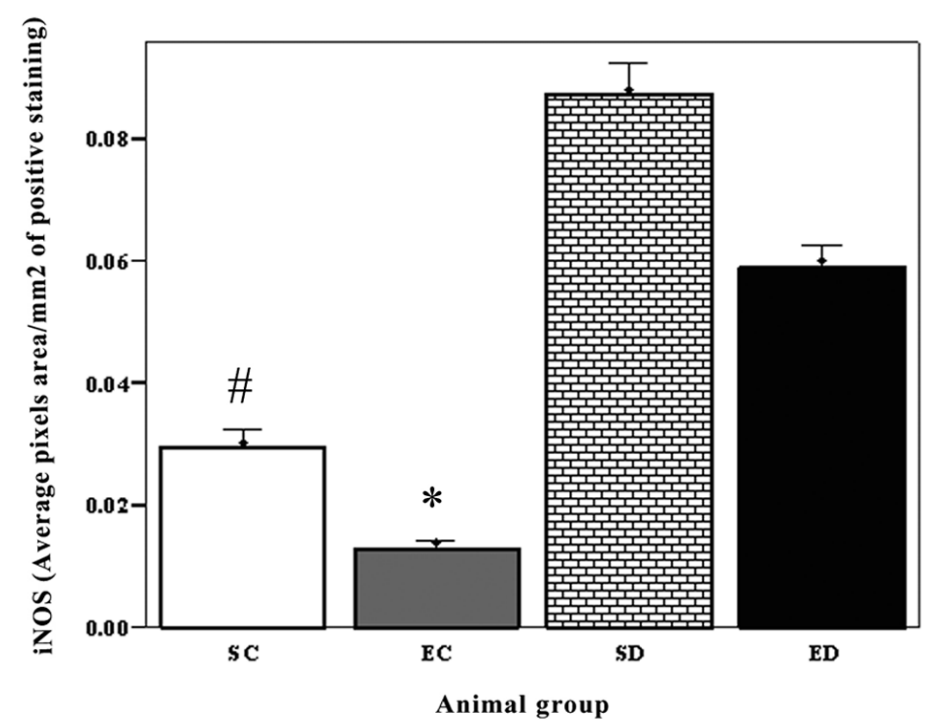

Figure 2. The expression of iNOS in cardiac muscle. The expression of iNOS showed significant increase in the sedentary diabetic rats compared to the sedentary control group $(P<0.03$, \#). There was a significant difference between exercise control and exercised diabetic group $(P<0.01)$. Exercise decreased the level of iNOS in the diabetic groups, but this decrease was not significant $(P<0.07)$. SC: Sedentary Control. EC: Exercised Control. SD:Sedentary Diabetic. ED: Exercise Diabetic.

the endogenous peroxidase activity for 5 minutes and then were washed in phosphate buffered saline (PBS). According to the manufacturer's instructions, sections were incubated for 1 hour at room temperature with P53 (Santa Cruz Biotechnology, Santa Cruz, CA) and iNOS (Biocare Medical, San Antonio, TX) antibodies which were diluted according to vendor instructions. Subsequently sections were washed in PBS and incubated with biotinylated secondary antibody (LSAB kit, Dako Carpinteria, CA) for 15 minutes at room temperature, then washed with PBS. Sections were incubated with streptavidin horse radish peroxidase (LSAB kit, Dako) for 15 minutes at room temperature and washed with PBS, 3, 3'-Diaminobenzidine (DAB) substrate was applied for 2 minutes or longer, until the desired color intensity was developed, and then the slides were washed with tap water to stop the reaction. Omission of the primary antibody from these samples served as negative controls. All sections were counterstained with hematoxylin and examined by light microscopy. Ten slides from each animal group were evaluated for $\mathrm{p} 53$ and iNOS expression in the left ventricle.

\section{Data analysis}

One way ANOVA was completed on all animals followed by paired and unpaired student t-test analysis to determine statistical significance within each group and to compare two groups. The area labeled with specific antibody and the percentage of cells positive for the antigen was calculated using published procedures $[15,16]$. Differences at $\mathrm{p}$ value $<0.05$ were considered statistically significant.

\section{Results}

Levels of both iNOS and p53 increased in the heart of diabetic rats. The expression level of $\mathrm{p} 53$ increased significantly in the diabetic sedentary group compared to sedentary control groups $(\mathrm{P}<0.02, \#)$. Exercise training decreased the expression level of P53 $(\mathrm{P}<0.005)$ (Fig. 1). The expression of iNOS showed significant increase in the sedentary diabetic rats compared to the sedentary control group ( $\mathrm{P}<0.03$, \#). There was a significant difference between exercise control and exercised diabetic group $(\mathrm{P}<0.01)$. Exercise decreased the level of iNOS in the diabetic groups, but this decrease was not significant $(\mathrm{P}<0.07)$ (Fig. 2).

\section{Discussion}

Diabetes mellitus has been shown to be associated with several cardiovascular complications including atherosclerosis, cardiac autonomic neuropathy, as well as cardiac myopathy [2]. These complications lead to increased morbidity and mortality rate among these patients [1]. Nitric oxide (NO) is a gaseous free radical that is generated under the influence of nitric oxide synthase (NOS) and has been proven to plays an integral role in several physiological activities in cell-cell communication, cellular defense, and cell injury [17] as well as being an intermediate in the pathology of several systemic diseases including cardiovascular diseases and both types of diabetes mellitus [18].

In this study, there was a marked increase in iNOS ex- 
pression in the hearts of both sedentary and exercise rats. In support of these findings, Ceriello et al have demonstrated that hyperglycemia induced iNOS gene expression in the hearts of studied rats [19]. Accordingly, the induction of iNOS expression suggests an increased release of NO that in turn increase the oxidative damage on cardiac myocytes mediated by the generation of peroxynitrite and other reactive nitrogen species [20]. NO production has been shown to be associated with contractile dysfunction and subsequently death of cardiac myocytes [21]. In support to that, Dias et al have reported that the iNOS expression in cardiac myocytes is induced following pathological situations where it contributes to myocardial dysfunction [22].

Cosenzi et al have shown that following to the induction of diabetes in rats, iNOS is activated in their kidney which is suggestive of the protective role of NO observed in diabetes where NO may be involved in inducing glomerular filtration observed in diabetic patients [23].

Apparently, the protective role of iNOS expression on the development and progression of certain pathological settings is context specific where in cardiovascular disorders it is deleterious and may result in death of cardiac mycoytes. Therefore, to investigate the survival of cardiac myocytes, the expression of p53 was examined and results showed a significant induction of p53 expression in the heart of sedentary diabetic rats compared to sedentary control rats $(\mathrm{P}<$ 0.05). Considering the role of p53 in initiating programmed cell death (apoptosis), these findings indicates that hyperglycemia in diabetic rats indicates apoptotic death of myocytes and is, therefore, supportive to the possible harmful rather than protective effect of iNOS on myocytes in response to hyperglycemia.

In this study, we aimed to investigate the effect of exercise on reducing the risk of developing cardiovascular complications, specifically cardiac myopathy among diabetic rats. It is well established that exercise are advantageous to type II diabetic patients and associated with effective control of hyperglycemia [24]. While in type I disease, patients should follow a treatment regimen that consider a combination of exercise, dietary pattern as well as insulin therapy to avoid the risk of hypoglycemia [24, 25]. Herein, we have shown that the exercised diabetic rats were having a significant reduced expression of iNOS in their hearts compared to sedentary animals $(\mathrm{P}<0.05)$. Exercise has an effect on glucose and nutrient metabolism and accordingly on the results in a better control of hyperglycemia in both type I and type II diabetes. Subsequent to the exercise-mediated control of glucose levels, iNOS levels are reduced $(\mathrm{P}<0.07)$. This may suggest a beneficial effect of exercise in reducing cardiovascular complications mediated by the release of NO in cardiac tissues. P53 levels in rats following to exercise were determined and data have shown a significant reduction in its expression in cardiac myocytes of diabetic rats compared to sedentary diabetic rats $(\mathrm{P}<0.005)$ indicating a highly ef- fective reduction in apoptosis of cardiac myocytes even to the slight non-significant reduction of iNOS expression. This significant reduction in p53 versus non-significant reduction in iNOS may suggest that exercise may also interfere with the reduction of p53 through other pathways that ultimately improve the survival of cardiac mycoytes.

In conclusion, our data suggests that exercise has positive impact on reducing the risk of cardiac myopathy mediated by hyperglycemia in type I diabetes.

\section{Acknowledgement}

This study was financially supported by The Deanship of Research at Jordan University of Science and Technology. Irbid, Jordan.

\section{References}

1. Scheuermann-Freestone M, Madsen PL, Manners D, Blamire AM, Buckingham RE, Styles P, Radda GK, et al. Abnormal cardiac and skeletal muscle energy metabolism in patients with type 2 diabetes. Circulation. 2003;107(24):3040-3046.

2. Yudkin JS. Hyperglycaemia as a cardiovascular risk factor in diabetes. Lancet. 2008;372(9643):1036-1037.

3. Howarth FC, Shafiullah M, Qureshi MA. Chronic effects of type 2 diabetes mellitus on cardiac muscle contraction in the Goto-Kakizaki rat. Exp Physiol. 2007;92(6):10291036.

4. Shen X, Zheng S, Thongboonkerd V, Xu M, Pierce WM, Jr., Klein JB, Epstein PN. Cardiac mitochondrial damage and biogenesis in a chronic model of type 1 diabetes. Am J Physiol Endocrinol Metab. 2004;287(5):E896905.

5. Low PA, Nickander KK, Tritschler HJ. The roles of oxidative stress and antioxidant treatment in experimental diabetic neuropathy. Diabetes. 1997;46 Suppl 2:S38-42.

6. Chen Z, Siu B, Ho YS, Vincent R, Chua CC, Hamdy RC, Chua BH. Overexpression of MnSOD protects against myocardial ischemia/reperfusion injury in transgenic mice. J Mol Cell Cardiol. 1998;30(11):2281-2289.

7. Kaul N, Siveski-Iliskovic N, Hill M, Khaper N, Seneviratne C, Singal PK. Probucol treatment reverses antioxidant and functional deficit in diabetic cardiomyopathy. Mol Cell Biochem. 1996;160-161:283-288.

8. Eeg-Olofsson K, Cederholm J, Nilsson PM, Zethelius B, Svensson AM, Gudbjornsdottir S, Eliasson B. Glycemic control and cardiovascular disease in 7,454 patients with type 1 diabetes: an observational study from the Swedish National Diabetes Register (NDR). Diabetes Care. 2010;33(7):1640-1646.

9. Reddy HK, Koshy SK, Wasson S, Quan EE, Pagni S, 
Roberts AM, Joshua IG, et al. Adaptive-outward and maladaptive-inward arterial remodeling measured by intravascular ultrasound in hyperhomocysteinemia and diabetes. J Cardiovasc Pharmacol Ther. 2006;11(1):6576.

10. Movahed MR, Hashemzadeh M, Jamal MM. Increased prevalence of third-degree atrioventricular block in patients with type II diabetes mellitus. Chest. 2005;128(4):2611-2614.

11. Habib S, Ali A. Biochemistry of nitric oxide. Indian $\mathrm{J}$ Clin Biochem. 2011;26(1):3-17.

12. Nakamura H, Matoba S, Iwai-Kanai E, Kimata M, Hoshino A, Nakaoka M, Katamura M, et al. p53 promotes cardiac dysfunction in diabetic mellitus caused by excessive mitochondrial respiration-mediated reactive oxygen species generation and lipid accumulation. Circ Heart Fail. 2012;5(1):106-115.

13. Fiordaliso F, Leri A, Cesselli D, Limana F, Safai B, Nadal-Ginard B, Anversa P, et al. Hyperglycemia activates p53 and p53-regulated genes leading to myocyte cell death. Diabetes. 2001;50(10):2363-2375.

14. Al-Jarrah M, Pothakos K, Novikova L, Smirnova IV, Kurz MJ, Stehno-Bittel L, Lau YS. Endurance exercise promotes cardiorespiratory rehabilitation without neurorestoration in the chronic mouse model of parkinsonism with severe neurodegeneration. Neuroscience. 2007;149(1):28-37.

15. Al-Jarrah M, Matalka I, Aseri HA, Mohtaseb A, Smirnova IV, Novikova L, Stehno-Bittel L, et al. Exercise training prevents endometrial hyperplasia and biomarkers for endometrial cancer in rat model of type 1 diabetes. $\mathrm{J}$ Clin Med Res. 2010;2(5):207-214.

16. Al-Jarrah MD, Jamous M. Effect of endurance exercise training on the expression of GFAP, S100B, and NSE in the striatum of chronic/progressive mouse model of Par- kinson's disease. NeuroRehabilitation. 2011;28(4):359363.

17. Raij L, Baylis C. Glomerular actions of nitric oxide. Kidney Int. 1995;48(1):20-32.

18. Soskic SS, Dobutovic BD, Sudar EM, Obradovic MM, Nikolic DM, Djordjevic JD, Radak DJ, et al. Regulation of Inducible Nitric Oxide Synthase (iNOS) and its Potential Role in Insulin Resistance, Diabetes and Heart Failure. Open Cardiovasc Med J. 2011;5:153-163.

19. Ceriello A, Quagliaro L, D’Amico M, Di Filippo C, Marfella R, Nappo F, Berrino L, et al. Acute hyperglycemia induces nitrotyrosine formation and apoptosis in perfused heart from rat. Diabetes. 2002;51(4):10761082.

20. Cheng X, Cheng XS, Kuo KH, Pang CC. Inhibition of iNOS augments cardiovascular action of noradrenaline in streptozotocin-induced diabetes. Cardiovasc Res. 2004;64(2):298-307.

21. Balligand JL, Cannon PJ. Nitric oxide synthases and cardiac muscle. Autocrine and paracrine influences. Arterioscler Thromb Vasc Biol. 1997;17(10):1846-1858.

22. Dias FA, Urboniene D, Yuzhakova MA, Biesiadecki BJ, Pena JR, Goldspink PH, Geenen DL, et al. Ablation of iNOS delays cardiac contractile dysfunction in chronic hypertension. Front Biosci (Elite Ed). 2010;2:312-324.

23. Cosenzi A, Bernobich E, Bonavita M, Trevisan R, Bellini G, Campanacci L. Early effects of diabetes on inducible nitric oxide synthase in the kidney. Acta Diabetol. 2002;39(2):91-96.

24. De Feo P, Di Loreto C, Ranchelli A, Fatone C, Gambelunghe G, Lucidi P, Santeusanio F. Exercise and diabetes. Acta Biomed. 2006;77 Suppl 1:14-17.

25. Toni S, Reali MF, Barni F, Lenzi L, Festini F. Managing insulin therapy during exercise in type 1 diabetes mellitus. Acta Biomed. 2006;77 Suppl 1:34-40. 